\title{
The reciprocity theorem for porous anisotropic media
}

\author{
E. Boschi (*)
}

Received on July I7th, 1972

Sumakr. - In this paper we give a reciprocity theorem for anisotropic porous media in the quasi-stationary case. The distribution of the pores is assumed statistically homogeneous.

Riassuxro. --.. Viene stabilito un teorema di reciprociti per mezzi porosi anisotropi nel caso quasi-stazionario. Lat distribuzione dei pori i assunta statisticamente omogenea.

\section{1. - Imtroduction.}

The theory of deformation of porous materials containing a fluid has been reveloped by Biot $\left({ }^{1,2}\right)$, for the case of an elastic solid. In the last years various generalizations and particular applications have been consiclered.

A porous solid is representer as an elastic skeleton, having compressibility and shear rigidity, with a statistical distribution of interconnecter pores containing a compressible fluirl. It is understood that the term "porosity" refers to the effective porosity, namely, that encompassing only the intercommunicating voir spaces as opposed to those pores which are sealed off. In the following, the word "pore" will refer to the effective pores while the sealer pores will be considered as a part of the solid.

(*) Istituto di Fisica, Eniversiti di Bologna, Italia. 
This system of fluid and solid is a general elastic system with conservation properties. The deformation of a unit rube is assumed to be completely reversible.

By deformation is meant, here, that determined by both strain tensors in the solid and in the fluid phases.

Ieșan $\left({ }^{6}\right)$ has established a method by means of which he was able to give reciprocity theorems in the dynamic theory of continua, without using Laplace transforms in the case of nonhomogeneous initial conditions. Moreover, by this method, he obtained reciprocity relations which involve only the displacement vector and the known functions.

Boschi (3) has obtained a reciprocity theorem for isotropic porous homogeneous media in the quasi-stationary case. He $\left(^{4}\right)$ has also been able to give a variational theorem in the linear theory of porous merlia, and in the theory of viscoelastic porous merlia $\left(^{5}\right)$.

This kind of speculations is of great interest because they form the basis for the solution of problems arising in many diversified fields such as seepage in soil mechanies, ground water hydrology, petroleum engineering, water purifications, acoustic engineering and so on.

\section{2. - BASIC EQUATIONS.}

Throughout this paper we employ a rectangular coordinate system, $O x_{k}(k=1,2,3)$, and the usual inclicial notations. Let $\bar{V}$ be a regular (in the sense of Kellog) region of space occupied by an anisotropic porous solid, whose boundary is $\Sigma$. Moreover $V$ is the interior of $\bar{r}, n_{1}$ are the components of the unit outward normal to $\Sigma$.

For convenience and clarity in presentation, all regularity hypotheses on considered functions will be omitterl.

On these basis the field equations for anisotropic porous solids, in the qnasi-static case, are:

- the constitutive equations:

$$
\begin{aligned}
\sigma_{i j} & =C_{i j k l} e_{k l}+\alpha_{i j} \varepsilon \\
\sigma & =\alpha_{i j} e_{i j}+\beta \varepsilon
\end{aligned}
$$

- the equations of equilibrium:

$$
\left(\sigma_{i j}+\sigma \delta_{i j}\right)_{, \jmath}+\varrho F_{\imath}=0
$$


The Darev's law:

$$
\sigma, i+o_{1} F_{i}=b_{i j}\left(\dot{C}_{j}-\dot{u}_{j}\right) .
$$

The equation [2.b] is the Darcy's law in a generalizerl form for an anisotropic medlium.

- the strain-rlisplacement relations:

$$
\begin{gathered}
2 e_{i j}=u_{i, j}+u_{j, i} \\
\varepsilon=U_{i, j}
\end{gathered}
$$

In these equations we have used the following notations: $u_{i}$, the components of the displacement vector for the solid phase; $C_{i}$, the components of the displacement vector for the Huid inside the pores; $\sigma_{i j}, e_{i j}$, the components of the strain and of the stress tensors for the solid phase; $\sigma$, the hydrostatic state of stress of the liquid filling the pores, i.e., if we consider a cube of unit size of the bulk material, $\sigma$ represents the total normal tension force applied to the fluid part of the faces of such a cube; $\varepsilon_{i j}$, the components of the strain tensor for the fluid phase; $\varepsilon-\varepsilon_{i i}$, dilatation of the fluid; $\varrho$, $\varrho_{1}$ densities of the solid and of the fluid, respectively; $C_{i j h}, b_{i}$, characteristies of the solirl phase; $\alpha_{i j}, \beta$, characteristics of the fluirl phase $\left({ }^{2}\right)$. Let us also remember that a comma denotes partial derivation with respect to the space variables, $x_{k}$, and a dot denotes partial derivation with respect to the time $t$.

Furthermore the following symmetry relations:

$$
\begin{gathered}
C_{i j k l}=C_{j i k l}-C_{k i l i} \\
\alpha_{i j}=a_{j i} \\
b_{i j}=b_{j i}
\end{gathered}
$$

are satisfied.

To the system of the field equations we must arljoin the initial condlitions:

$$
\begin{aligned}
& u_{i}(x, 0)=u_{i} \\
& U_{i}(x, 0)=A_{i}
\end{aligned}
$$

and the boundary conditions:

$$
\left(\sigma_{i j}+\sigma \delta_{i j}\right) n_{j}=p_{i}, \quad \text { on } \Perp
$$

where $a_{i}, A_{i}, p_{i}$ are prescribed functions. 
3. - PRELIMINARIES.

Let $f, y$ and 7 be function on $\bar{V} \times[0, \infty)$, continuous on $[0, \infty)$ with respect to the time $t$ for each $x \in \bar{V}$. We denote by $f * g$ the convolution of $f$ and $q$ :

$$
[f * g](x, t)=\int_{i}^{\mathrm{t}} f(x, t-s) y(x, s) d s .
$$

We will have occasion to use the following well-known properties of the convolution $\left({ }^{9}\right)$ :

$$
\begin{gathered}
f * g=y * f \\
f *(g * h)=(f * g) * h-f * g * h \\
f *(g+h)=f * g+f * h .
\end{gathered}
$$

Henceforth we will denote by $l$ the function defined on $[0, \infty)$ by:

$$
l(t)=1 .
$$

It is easy to show that:

$$
\begin{aligned}
& l *\left(U_{j}-u_{j}\right)=U_{j}-u_{j}-\left(A_{j}-a_{\jmath}\right) \\
& l *\left(\sigma, l+\varrho_{1} F_{l}\right)=l * \sigma, i+\varrho_{1} l * F_{l} .
\end{aligned}
$$

Then from the equations [2.b], [7] and [8], we get:

$$
l * \sigma, i+q_{i}=b_{i j}\left(U_{j}-u_{j}\right)
$$

where:

$$
q_{1}=\varrho_{1} l * F_{i}+b_{l j}\left(A_{j}-a_{j}\right) \text {. }
$$

The equations [9] are equivalent to the equations [2.b] and to the initial conditions [5.a], [5.b].

\section{4. - THE RECIPROCITY THEOREM.}

Let us now consider the body subjected to two different systems of elastic loarlings:

$$
L^{(\alpha)}=\left\{F_{i}{ }^{(\alpha)}, p_{i}{ }^{(\alpha)}, A_{i}{ }^{(\alpha)}, a_{i}{ }^{(\alpha)},\right\} \quad \alpha=1,2 .
$$


The two corresponding configurations are:

$$
C^{(\alpha)} \equiv\left\{U_{i}^{(\alpha)}, u_{i}^{(\alpha)}, \rho_{i j}{ }^{(\alpha)}, \varepsilon^{(\alpha)}, \sigma_{i j}^{(\alpha)}, \sigma^{(\alpha)},\right\} \quad \alpha=1,2 .
$$

From the equations [1.a], [1.b], we get:

$$
\begin{aligned}
& \sigma_{i j}^{(\alpha)}-\alpha_{i j} \varepsilon^{(\alpha)}=C_{i j k l} e_{k \cdot l}^{(\alpha)} \\
& \sigma^{(\alpha)}-\alpha_{i j} \varepsilon^{(\alpha)}=\beta \varepsilon^{(\alpha)}
\end{aligned} \quad\{a=1.2
$$

It is true that

$$
\begin{aligned}
& \left(\sigma_{i j}^{(1)}-\alpha_{i j} \varepsilon^{(1)}\right) * e_{i j}^{(2)}=C_{i j k l} e_{k-l^{(1)}} * e_{i j}^{(2)} \\
& \left(\sigma_{i j}^{(2)}-\alpha_{i j} \varepsilon^{(2)}\right) * e_{i j}^{(1)}=C_{i j k l} e_{k l^{(2)}} * e_{i j}^{(1)}
\end{aligned}
$$

Adding these two relations, we have:

$$
\left(\sigma_{i j}(1)-\alpha_{i j} \varepsilon^{(1)}\right) * e_{i j}^{(2)}=\left(\sigma_{i j}^{(2)}-\alpha_{i j} \varepsilon^{(2)}\right) * e_{i j}^{(1)} .
$$

It is also true that:

$$
\begin{aligned}
& \left(\sigma^{(1)}-a_{i j} e_{i j}^{(1)}\right) * \varepsilon^{(2)}=\hat{\rho} \varepsilon^{(1)} * \varepsilon^{(2)} \\
& \left(\sigma^{(2)}-a_{i j} e_{i j}^{(2)}\right) * \varepsilon^{(1)}=\beta \varepsilon^{(2)} * \varepsilon^{(1)}
\end{aligned}
$$

and, by addition, we obtain:

$$
\left(\sigma^{(1)}-\alpha_{i j} e_{i j}^{(1)}\right) * \varepsilon^{(2)}=\left(\sigma^{(2)}-\alpha_{i j} e_{i j}^{(2)}\right) * \varepsilon^{(1)} .
$$

Adding the relations [13] and [1f] we get:

$$
\sigma_{i j}^{(1)} * e_{i j}^{(2)}+\sigma^{(1)} * \varepsilon^{(2)}=\sigma_{i j}^{(2)} * e_{i j}^{(1)}+\sigma^{(2)} * \varepsilon^{(1)} .
$$

If we introduce the notation:

$$
I_{\alpha \beta}=\int_{j} l *\left(\sigma_{i j}{ }^{(\alpha)} * e_{i j}(\hat{\beta})+\sigma^{(\alpha)} * \varepsilon^{(\beta)}\right) d V ; \quad \alpha, \stackrel{\rho}{\nu}-1,2
$$

from equation [15] we have:

$$
I_{12}=I_{21}
$$


Using the relations $[3],[9],[10]$ and the divergence theorem, we get:

$$
\begin{aligned}
& I_{\alpha \beta}=\int_{\underline{\Sigma}} l * p^{(\alpha)} * u_{i}^{(\beta)} d \Sigma+\int_{\Sigma} l * \sigma^{(\alpha)} * U_{i}^{(\beta)} m_{i} d \Sigma+ \\
& \div \int_{V} l * \underline{0} F_{i}^{(\alpha)} * u_{i}^{(\beta)} d V-\int_{V} l * \sigma^{(\alpha)} * \rho^{i \beta)} d V- \\
& -\int_{V^{\circ}} b_{i j}\left(V_{j}^{(\alpha)}-\mu_{j}^{(\alpha)}\right) * C_{i}^{(\beta)} d V+\int_{V^{-}} q_{i}^{(\alpha)} * V_{i}^{(\beta)} d V, \\
& \alpha, \beta=1, \geq
\end{aligned}
$$

Finally from equations [17] and [18] we can state the reciprocity theorem for anisotropic porous solids:

If an anisotropic porous solid is subjected to two different systems of elastic loadings [11], then between the two corresponding configurations [12] there is the following reciprocity relation:

$$
\begin{aligned}
& \int_{\Sigma} l * p_{i}^{(1)} * u_{i}^{(2)} d I+\int_{\Sigma} l * \sigma^{(1)} n_{i} * U_{i}^{(2)} d I+ \\
& +\int_{V} l \varrho * F_{i}^{(1)} * u_{i}^{(2)} d V-\int_{V} l * \sigma^{(1)} * e^{(2)} d V- \\
& -\int_{V}\left[b_{i j}\left(U_{j}^{(1)}-u_{j}^{(1)}\right)+q_{i}^{(1)}\right] * U_{v^{(2)}} d V= \\
& =\int_{\Sigma} l p_{i}^{(2)} * u_{i}^{(1)} d \Sigma+\int_{\Sigma} l * \sigma^{(2)} u_{i} * U_{i}^{(1)} d \Sigma+ \\
& +\int_{V} l * \varrho F^{(2)} * u^{(1)} d V^{r}-\int_{V} l * \sigma^{(2)} * e^{(1)} d V- \\
& --\int_{V}\left[b_{i j}\left(U_{j}^{(2)}-u_{j}^{(2)}\right)+\Psi^{(2)}\right] * U_{i}^{(1)} d Y .
\end{aligned}
$$

We should want to put in evidence that, throughout this paper, we did not use any restrictive assumption. The theorem [19] is valid under very general conditions. 
In the special case of homogeneous boundary conditions, of great interest in the applications, the relation [19] reduces to:

$$
\begin{aligned}
& \int_{V}\left\{l *\left(\varrho F_{i}^{(1)} * u_{i}^{(2)}-\sigma^{(1)} * e^{(2)}\right)-\left[b_{i j}\left(U_{j}^{(1)}-u_{j}^{(1)}\right)+q_{i}^{(1)}\right\} * U_{i}^{(2)}\right\} d V= \\
& =\int_{V}\left\{l *\left(\varrho \bar{F}^{(2)} * u_{i}^{(1)}-\sigma^{(2)} * e^{(1)}\right)-\left[b_{i j}\left(U_{j}^{(2)}-u_{j}^{(2)}\right)+q^{(2)}\right] * U_{i}^{(1)}\right\} d V .
\end{aligned}
$$

The reciprocity theorem derived in this paper can be used to obtain variational theorems in the theory of porous anisotropic materials. These theorems will be developed in another paper.

\section{REFE RENCES}

(1) Biot M. A., 1955. - “J. Appl. Phys.", 26, 182.

$\left({ }^{2}\right)$ Biot M. A., 1956. - "J. Appl. Phys.", 27, 240.

(3) Boschi E., 1972. - "Lett. Nuovo Cimento", 4, 973.

(4) Boscir E., 1972. - To be published.

(5) Boschi E., 1972. - To be published.

$\left.{ }^{(}\right)$IEșAn D., 1967. - "Rend. Acad. Sc.", Paris, 265, 271.

(7) Ieșan D., 1969. - "Int. J. Engn. Sci.", 7, 1213.

(8) Ieșan D., 1970. - "Rev. Roum. Math. Pures et Appl.", 15, 1181.

$\left({ }^{9}\right)$ Mikusinski J., 1959. - Operational Calculus, Pergamon Press, New York. 